\title{
Winnicott hoy día: Consideraciones psicoanalíticas acerca de las nuevas Bases Técnicas del Sename sobre adopción
}

\author{
Winnicott Today: Psychoanalytic Considerations About \\ New Sename's Technical Basis for Adoption
}

Leonardo Medeiros Ruiz ${ }^{1}$

\section{Resumen}

Se discuten las nuevas Bases Técnicas del Servicio Nacional de Menores (Sename) sobre políticas de adopción en la infancia, desde el pensamiento del psicoanalista Donald Winnicott. Se plantea que la teoría y clínica winnicottianas no se comprenden bien si no se tiene en cuenta su ética, que subraya el deseo e historia del nińo y sus padres. Las consecuencias de esta posición son relevantes como instrumento de análisis crítico en tanto permite desentrañar la noción de infancia que fundamenta tales bases técnicas. Adicionalmente, este trabajo revitaliza las concepciones del psicoanalista inglés, al mostrar que su lectura aún puede iluminar nuestra manera de pensar las problemáticas de la infancia, incluso si estas se circunscriben a la realidad chilena actual.

Palabras clave: Winnicott, clínica, ética, politicas adopción, Sename.

\section{Abstract}

The new Technical Basis of Servicio Nacional de Menores (Minor National Services, Sename) regarding adoption politics in infancy are discussed in relation to Donald Winnicott's theory. It is stated that winnicottian theory and clinic are not well understood if its ethics is not kept in mind, as it underlines the wish and history of the child and parents. The consecuences of this position are relevant

1 Psicólogo Centro de Asistencia a Víctimas de Atentados Sexuales (CAVAS). Santiago de Chile. E-mail: leomedeirosruiz@yahoo.com 
as instruments of critical analysis, as they unravel the notion of infancy that sustains such Technical Basis. This paper also revitalizes Winnicott's thoughts, as reading his work can still enlighten our way of thinking about infancy problems, even if these are circumscribed to current chilean context.

Key words: Winnicott, clinical, ethics, adoption policies, Sename.

\section{Planteamiento del problema}

Este trabajo ensaya un análisis crítico a las nuevas Bases Técnicas sobre Adopción del Servicio Nacional de Menores, en particular las nociones de historia y de infancia implícitas en él. Según lo señalado por estas bases, su propuesta surge en respuesta a dificultades diagnosticadas del funcionamiento por casi tres años de los "Programas de reparación del abandono para la integración de niños/as institucionalizados en familias alternativas a la de origen" (Sename, 2011, p. 2), implementados durante el año 2008 en las regiones Metropolitana, Valparaíso, Biobío y de la Araucanía, y cuya oferta programática solo consideraba alternativas de atención frente a situaciones graves de vulneración como situaciones de calle, consumo de drogas o maltrato grave, pero no para situaciones de abandono e inhabilidad parental. Las dificultades diagnosticadas dicen relación con el modelo terapéutico y los extensos tiempos del tratamiento. En cuanto a lo primero, se insiste en la ausencia de objetivos específicos de acuerdo al nivel de desarrollo evolutivo del niño, teniendo como objetivo general solo la integración y elaboración de las experiencias de vida. Entonces, una de las primeras cuestiones que estipulan estas bases, es el establecimiento de objetivos e intervenciones terapéuticos diferenciados de acuerdo a rangos etarios, cuyas definiciones se extraen de investigaciones empíricas basadas en la evidencia. Este empeño resultaría controversial en tanto se sustenta en una concepción del déficit del niño pequeño en su capacidad de historización y simbolización de sus experiencias pasadas (Sename, 2011). 
Las políticas de adopción son una respuesta posible a las problemáticas concernientes a las graves vulneraciones de derecho en la infancia, cuando irremediablemente el Estado interviene para separar al niño de su familia de origen. Sin embargo, estas mismas políticas deben asegurar que sus intervenciones a la realidad del niño, no le infringirán una nueva violencia que traiga consecuencias nefastas para su desarrollo afectivo. Es en este sentido que estas bases, guiadas por principios extraídos de la psicología conductual y cognitivista, y, siguiendo una terminología donde dominan conceptos como "entrenamiento", "auto-regulación” y "estrés", resultan debatibles desde otras perspectivas teóricas que contemplan concepciones del niño y de la infancia distintas, que tal vez pudieran dialogar y arrojar luces, también teniendo como horizonte el "bienestar superior del niño". Una de las cuestiones que interesa develar es cómo ciertos conceptos winnicottianos, muy popularizados, como el de "objeto transicional" es subsumido y reinterpretado por estas bases, para darle una dimensión técnica y de entrenamiento cuyo objetivo será combatir el malestar o la angustia del niño -y la del adulto cuidador, principalmente-, reprimiéndose su verdadera raíz clínica y ética.

Es en este contexto que interesa situarse desde el pensamiento de Winnicott y especialmente sobre lo que pudo plantear acerca de la adopción, desarrollos que puedan permitirnos contrastar con los principios teóricos y éticos que sustentan las Bases Técnicas. Una de las consecuencias de esta re-visión sobre el pensamiento de Winnicott es un trabajo con el autor, el que finalmente promete acercarnos a ceñir su rasgo propio, en tanto persona y psicoanalista.

Al contrario de lo que pudieran sugerir estas bases técnicas, las concepciones y teorías winnicottianas se desprenden de lo que puede llamarse, más ampliamente, su ética; entendiendo por esta, preliminarmente, el cómo Winnicott se ubica como psicoanalista frente a las problemáticas sociales y políticas de su época, en particular las que conciernen a la infancia, cuestión que se desprende no solo de su labor de psicoanalista en el consultorio, donde encontramos testimonio de ello en sus escritos y conferencias, sino también de sus intervenciones dirigidas a los no analistas 
sobre temas de actualidad. Ejemplo de ello son sus escritos que tratan sobre la cuestión del desamparo de los niños que quedaban huérfanos durante la segunda guerra mundial, y que debían ser reubicados en hogares sustitutos (Winnicott, 2007a, 2007b) cuestión que será tratada más adelante en este artículo.

Sin embargo, cuando intentamos acercarnos a la clínica winnicottiana, él mismo nos alerta sobre la dificultad de poder transmitirla, puesto que esta de ninguna manera podrá ser reducible a un asunto puramente técnico, el cual se definiría por ser homogeneizable. Por eso opta por el relato de casos, modo de transmisión del saber psicoanalítico enseñado por Freud, y que pareciera ajustarse mejor a su objeto de estudio lo inconsciente-, que se define, en primer lugar, por su singularidad. Sobre esto se expresa Winnicott en la introducción a su "Clínica psicoanalítica infantil" (Winnicott, 2008), que trata sobre las primeras entrevistas en la consulta de psiquiatría infantil: "Difícilmente pueda llamarse técnica al modo de operar en este trabajo. No existen dos casos iguales, y entre el terapeuta y el paciente se da un intercambio mucho más libre que el que se produce en un tratamiento psicoanalítico ortodoxo" (Winnicott, 2008, p. 9). Él introduce el placer del lector como criterio de la calidad de su trabajo, es decir, le interesa saber si su escritura da cuenta verdaderamente del caso. Se apoya en la metáfora musical:

(...) el criterio con que han de medirse las presentes descripciones de los casos debe descansar en el grado de placer puesto de manifiesto en la lectura. Si esta se hace trabajosa, significará que he sido demasiado artificioso: me dediqué a hacer gala de la técnica y no a interpretar la música (Winnicott, 2008, p. 9).

Primera indicación entonces sobre la ética: que el psicoterapeuta haga la experiencia de que la técnica es solo una herramienta útil que permite operar en el trabajo analítico. Sin embargo, la técnica no transmite nada del análisis ni del deseo del analista. Este deseo no puede consistir sino en que el paciente encuentre su manera de ser, así como el terapeuta encontrará su manera de trabajar y de transmitir a otros su trabajo. Aquí Winnicott otra vez nos desalienta, ya que se engaña el analista si confía demasiado en los registros de sesión para transmitir fielmente la experiencia del análisis: 
(...) es sabido que es difícil informar con exactitud. Ni la grabación en cinta magnetofónica ni el video pueden dar solución a este problema (...) buena parte de una entrevista y, en especial, sus detalles más sutiles, se borra como un sueño al nacer el día (Winnicott, 2008, p. 11).

Este pasaje muestra el reconocimiento de Winnicott de la acción de la represión para estropear la exactitud del relato, pero al mismo tiempo se la valora como la pieza que otorga singularidad al caso, haciéndolo -paradójicamente- mejor legible.

¿De qué se trata esta pieza propia al análisis que determina su dificultad de transmisión, y que al mismo tiempo es lo esencial en lo que concierne al deseo y la ética de Winnicott? Pienso que esta pregunta acaso permite acercarnos a la que considero su contribución más importante al psicoanálisis, se trata de la introducción de la cuestión del ser en el pensar psicoanalitico, cuestión de raíz filosófica que encuentra traducción clínica en lo que él denominó self. El self no es el yo freudiano, puesto que este se encuentra capturado en el conflicto psíquico. Antes que eso, el self dialoga mejor con el sujeto lacaniano, con la salvedad de no restringirlo a una teoría formalista del significante; aproximación de Winnicott a Lacan, porque su pensamiento gira en torno al sujeto del deseo, rompiendo el centro de gravedad del psicoanálisis cuya hegemonía en Inglaterra se había entronizado alrededor del objeto de la pulsión, con el riesgo de cosificación al que ese ejercicio invita. Las coordenadas para aprehender el self winnicottiano habría que buscarlas en la concepción del objeto transicional: aquel objetosubjetivo que permite al niño hacer la experiencia de la primera posesión another than me, en otras palabras, de una primera existencia humana que es la introducción de una alteridad como siendo algo radicalmente nuevo en el desarrollo infantil. En definitiva, el self hablaría de esa primera experiencia y no de una categoría psicológica o de una tópica. De acuerdo a esto, podemos preguntarnos si lo esencial de la clínica winnicottiana no consiste en averiguar cómo y desde qué lugar el analista trabaja para facilitar en su paciente que esta experiencia del selfse constituya y luego se mantenga viva. 
Antecedentes teóricos

Es importante en este capítulo situar algunos conceptos winnicottianos, su origen y el contexto en que nacieron, y su diálogo y debates con otros autores (principalmente Melanie Klein y Jacques Lacan), que ayuden a encontrar el verdadero sentido que Winnicott quiso darles, para permitirnos luego discutir con fundamento en qué punto este autor se aleja de los principios que sustentan las Bases Técnicas. Específicamente, se abordan en profundidad las nociones de lo transicionaly el lugar del otro en la constitución subjetiva, que destronan la centralidad de la problemática del placer/ displacer en el psicoanálisis, y de paso cuestionan, por ejemplo, las clásicas dicotomías psicológicas de presencia/ausencia y de externo/interno.

Constatábamos que la ética winnicottiana está profundamente atravesada por el compromiso que asume el psicoanalista con las problemáticas de su época, de las que, quiéralo o no, toma una posición por el saber que encarna y el lugar institucional que ocupa, pero, ¿cómo se puede llegar a plantear una clínica y una ética winnicottiana a partir de sus escritos, los cuales despistan al lector por su aparente ligereza o desprolijidad conceptual, como se lo ha intentado etiquetar? Y un poco más allá, ¿cómo entiende Winnicott el psicoanálisis, y en particular el psicoanálisis infantil?

No hay que olvidar que Winnicott llega al psicoanálisis por el ejercicio de la pediatría, disciplina que le proporcionó la oportunidad de observar directamente al nińo y su familia. Se dio cuenta que el pediatra es demandado no solo en lo que se refiere a los cuidados físicos del niño, sino que también respecto de padecimientos graves como el autismo. Decide entonces no refugiarse en estandarizaciones sobre qué función cognitiva o psíquica debe alcanzar el niño y a qué edad, sino que se preocupa por investigar sobre el cómo el niño alcanza el campo del otro humano, es decir, cómo nace la preocupación por el otro en el niño pequeño. Lo que primero puede constatar, es que esta capacidad no puede alcanzarse sin la intervención del adulto que lo sostiene, a condición de asumir este mismo una posición muy singular la cual en adelante será objeto de estudio y pieza angular en sus escritos sobre psicoanálisis infantil. Primera tarea del bebé entonces: tiene que crear, con la ayuda del otro, un campo subjetivo. 
Lo que interesa señalar es el desarrollo que hace Winnicott sobre el problema del ser y su constitución, más que la pulsión y sus destinos, tema recurrente en el psicoanálisis infantil de raigambre kleiniana, y que había tenido su primera piedra con el análisis freudiano del caso del pequeño Hans. En este punto Winnicott no vacila: "Hay que ver, más allá del 'mito psicoanalítico' (que hoy por suerte está despareciendo), que la temprana infancia es una etapa de satisfacciones vinculadas a la erogeneidad oral" (Winnicott, 1998b, p. 263).

En efecto, lo que importará para Winnicott será lo que él llama el "uso del objeto", más que las satisfacciones que este puede aportar. Hay una paradoja que el niño -y en primer lugar quien hace la función materna- debe poder sostener: "es la paradoja y la aceptación de la paradoja: el bebé crea el objeto, pero éste ya estaba ahí, esperando que se lo crease y que se lo denominara objeto catectizado" (Winnicott, 1991a, p. 266). De modo que la primera tarea hacia una subjetividad en la infancia, es la posibilidad que al niño se le ofrece -incluso se lo impulsa- para crear su primera posesión; la condición es la existencia de la ilusión que puede experimentar el niño de él mismo haber creado un objeto subjetivo, vía obligada para acceder a un mundo que tome por real. De manera muy clara Winnicott transmite que su interés es el estudio de cómo el niño se apropia de una subjetividad -y por lo tanto, de las condiciones para la construcción de una historia propia-, que no es estrictamente ni el mundo interno ni el mundo externo: una de las dicotomías clásicas que la noción de lo transicional hace superar.

Esta conceptualización de lo que podemos llamar primeros tiempos de la infancia ${ }^{2}$ puede mantener un diálogo fructífero con Jacques Lacan, esto es corroborado por el hecho de que Winnicott escribe en el año 1967 un artículo titulado "Papel del espejo de la madre y la familia en el desarrollo del nińo" (Winnicott, 2007c) en respuesta al conocido "Estadio

2 En alusión a nuestras coincidencias con el trabajo de Marta González (González, 2010), que teoriza sobre el tránsito del niño pequeño de lo que son sus movimientos corporales y la utilización del espacio en la sesión, hasta la posibilidad del recuerdo. Es el adulto -destinatario de esta actividad del niñoquien le ofrece alojamiento en una cierta historia mítica, pero es el niño quien en adelante deberá hacer su propio trabajo de subjetivación, es decir, de re-traducir para sí mismo esa historia como siendo su primera posesión. 
del espejo" lacaniano (Lacan, 2003). Además de reconocer la contribución de Lacan, la tesis winnicottiana es fuerte: "el precursor del espejo es el rostro de la madre" (Winnicott, 2007c, p. 147), y de esta relación depende, en gran medida, la supervivencia psíquica del niño, el modo de tramitar las primeras angustias, que para Winnicott siempre son psicóticas por su cualidad de amenazas de desintegración yoica, estructuralmente distintas a las angustias edípicas derivadas de la amenaza de castración. Para entender estas angustias de tipo psicótico podemos recurrir al self, concepto central en Winnicott, y el término que él introduce como personalización (Winnicott, 1991b) -una versión positiva de la despersonalización-, para acercársele. Porque el self es un concepto que ayuda a graficar el anudamiento entre la psique y el cuerpo, y lo que es fundamental, la idea de una residencia en un cuerpo subjetivable, logro del niño que le otorga ese impulso a existir, del que nos habla Winnicott como criterio de salud.

La simpatía entre estos dos psicoanalistas podría explicarse porque compartirían la idea lacaniana del cuerpo fragmentado del infante, de la cual "el estadio del espejo es un drama cuyo empuje interno se precipita de la insuficiencia a la anticipación" (Lacan, 2003, p. 147), dada la condición inicial de prematuración de la cría humana. Anticipación que en todo caso da como consecuencia la primera enajenación del deseo en el deseo del Otro, pero al mismo tiempo otorga una identificación fundamental por permitirle al sujeto reconocerse en este gran Otro, de ahí su función subjetivante. La respuesta de Winnicott a Lacan avanza para ubicar esa función especular y estructurante en el rostro de la madre, es decir, aquella que puede devolverle al niño una experiencia integrada y no fragmentada, desde una posición de ir fallando paulatinamente: en clave lacaniana, es la madre como siendo no-toda, es decir, nunca una presencia absoluta.

Lacan se había interesado en el trabajo de Winnicott al punto de impulsar y finalmente concretar la traducción al francés de su artículo sobre los Objetos Transicionales en la revista La Psychoanalyse $e^{3}$. Años más tarde, cuando dicta su seminario sobre El Acto Analitico (Lacan, 1967-68),

3 La referencia de este saludo a Lacan puede encontrarse en una carta que le escribiera, fechada el 11 de febrero de 1960 (Winnicott, 1990). 
en la clase del 6 de diciembre de 1967, se refiere a Winnicott como un precursor de su propio pensamiento:

Es muy destacable este autor al que debemos uno de los más finos descubrimientos, me recuerda, y nunca dejará de volver a mis recuerdos en homenaje, la ayuda que el objeto transicional, como él lo llamó, me aportó cuando yo me interrogaba sobre la forma de desmitificar esa función del objeto llamado parcial tal como la vemos sostener para soportar la teoría más abstrusa, la más mitificante, la menos clínica sobre las pretendidas relaciones desarrollantes de lo pre-genital con relación a lo genital (Lacan, 1967-1968, p. 26).

Y un poco más adelante:

Basta con leer a Winnicott para traducirlo. Está claro que de algún modo, es fácil ver acá la relación de ese pedacito de trapo, ese pedacito de sábana, ese cachito manchado al que se aferra el niño con ese primer objeto de goce (...) (Lacan, 1967-1968, p. 26).

Claramente Lacan reconoce en Winnicott una de sus inspiraciones para inventar su objeto $a$. Sin embargo, no estamos en acuerdo totalmente con Lacan cuando sintetiza el objeto transicional como una función del objeto parcial, pues nos parece que no solo simplifica la complejidad del pensamiento winnicottiano, sino que lo "estigmatiza" como objeto de goce, cuando Winnicott está pensando en otro registro, el de la función del objeto transicional en la construcción de la realidad del otro, del objeto como una primera posesión no-yo en la formación del self.

Si volvemos al relato winnicottiano, siempre reaparece la cuestión del ser, o del sentimiento de existencia, de "ser real", que parte del otro, en este caso, de la experiencia de integración que devuelve quien sostiene al niño, el "rostro" de la madre: "Cuando miro se me ve, y por lo tanto existo" (Winnicott, 2007c, p. 151). En este caso, "sostén" no es un término que uno deba entender unívocamente, es decir, en lo que se ha llamado a mi juicio en un sentido demasiado restrictivo "psicoterapia de apoyo", aquella que se centra en la "presencia" del analista; por el contrario, para Winnicott lo que el analista debe sostener o hacer holding, es exactamente la paradoja 
entre el ir y venir de una presencia-ausencia. Se trata que el adulto pueda "jugar" a la ausencia, estando presente. Tal vez la escena que mejor grafica esta singular manera de estar con, la ciñe Ricardo Rodulfo en su trabajo de lectura del pensamiento winnicottiano, se trata del niño pequeño jugando de espaldas a la madre:

Veamos las dos situaciones que Winnicott presenta y articula, haciendo de paso que la escena "existencial" fundamente la "técnica": se trata de la escena del análisis de adultos y la escena del niño pequeño jugando, dando la espalda. En ambos casos, una excesiva necesidad de ver todo el tiempo al otro se torna una dificultad a veces insuperable para la adquisición de la capacidad. Reconduce así la situación analítica a una escena de escritura originaria en la que el niño juega a solas, según se dice, en presencia de otro. En esta etapa donde aún se supone ser mirado, resulta esencial que el niño se sepa mirado, pero a la vez que no se trate de una mirada que Green llamaría intrusita y Winnicott interferencia, cuando no ataque (Rodulfo, 2009, p. 192).

Se entiende que Winnicott está pensando en una zona transicional más que en un objeto transicional. Ante todo lo transicional se trata de una experiencia-que se pudo tener o no-, y por qué no decirlo, de una primera inscripción donde se apuntala el primer sentimiento de existencia, si acaso pensamos que la condición para pensar la asunción de la subjetividad es que debe tener un lugar en el Otro. El problema clínico que acecha a Winnicott a partir de este modo de pensar el psicoanálisis es que lo traumático son todas las interferencias que no permitieron que el niño pequeńo tuviera esa experiencia plena de juego. Juego en tanto "playing"y no "game", pues este último designa más bien las reglas estereotipadas que ordenan un juego, mientras que "playing", es el juego que se desarrolla libremente, que está teniendo lugar, según la distinción que propone Pontalis en su prólogo de Realidad y juego (Winnicott, 2007c). Es más, este autor nos ofrece una clave de lectura a este texto al señalar que: "Se podría afirmar sin excederse, que todo el libro está destinado a que el lector detecte dicha 'evidencia' y extraiga las consecuencias" (Winnicott, 2007c, p. 2). 
Podemos preguntarnos hasta qué punto el pensamiento de Winnicott nace a partir de una respuesta a una cierta manera de entender el psicoanálisis que es impulsado por Melanie Klein, puesto que ambos autores parecieran tener preocupaciones opuestas: mientras que para esta autora la interpretación ocupa un lugar central en el análisis -increíblemente, para no abandonar el dominio del complejo de Edipo, hace retroceder las pulsiones de destrucción a etapas primerísimas del desarrollo-, Winnicott se concentra en el trabajo de constitución subjetiva que el niño debe hacer. Sobre todo con su noción de "posición esquizo-paranoide", Klein investiga estados tempranos del psiquismo:

En mi criterio, el superyó -esa parte del yo que critica y controla los impulsos peligrosos, y que Freud ubicó en líneas generales en el quinto año de vida- actúa desde mucho antes. Según mi hipótesis, en el quinto o sexto mes después del nacimiento el bebé comienza a temer el daño que sus impulsos destructivos y su avidez podrían causar -o haber causado- a sus objetos amados (Klein, 2004, p. 259).

A esta postura paradigmática, Winnicott responde, para descentrar la preocupación del psicoanálisis por lo instintivo-genético: "en psicología, 'más profundo' no siempre significa 'más temprano'” (Winnicott, 1993, p. 231). Esta aseveración se diría incluso que alcanza el estatuto de interpretación -nos toca a nosotros hacer trabajar su sentido-; y por el contrario, subraya el marco (como función de límite y continuidad del self) en que se desenvuelve la terapia, que hacen posible la interpretación: "El psicoanálisis no consiste tan solo en interpretar el inconsciente reprimido; consiste más bien en proporcionar un marco profesional a la confianza, en el cual esa interpretación pueda llevarse a cabo" (Winnicott, 1994, p. 133).

Un poco más lejos, critica el estilo kleiniano -que no necesariamente es compartido por Klein misma- de la interpretación mecánica que algunas veces se hace percibir como propio a este círculo, las que tienen la finalidad de corroborar una construcción teórica, pero que reproducen el sometimiento del paciente en la transferencia. Winnicott también reconoce haber caído en un error tal, cuando declara: 
las interpretaciones dogmáticas dejan al niño solo dos posibilidades: una aceptación de lo que he dicho como propaganda o un rechazo de la interpretación, de mí y de toda la situación (...) los tratamientos psicoanalíticos prolongados me han llevado a ver que interpretaciones que parecían correctas hace diez años atrás, y que el paciente aceptaba por temor, resultaban ser, al final, defensas colusorias (Winnicott, 2008, p. 18).

Esta crítica de Winnicott a los kleinianos alcanza proporciones mayores. El problema de los discípulos es que, obnubilados por la admiración hacia el "maestro", se extravían de su propio deseo. En una carta a Melanie Klein, fechada el 17 de noviembre de 1952, le expresa esta preocupación:

Personalmente pienso que es muy importante que la obra suya sea reenunciada por personas que hagan los descubrimientos a su manera y que presenten lo que descubren en su propio lenguaje. Solo de este modo se mantendrá vivo el lenguaje. Si usted estipula que en el futuro solo su propio lenguaje debe ser utilizado para la enunciación de los descubrimientos de otra gente, el lenguaje se convertirá en un lenguaje muerto, como ya se ha convertido en la Sociedad (...) Usted es la única capaz de destruir este lenguaje denominado doctrina kleiniana y kleinismo y todo eso, con un propósito constructivo (Winnicott, 1990, pp. 89-90).

En efecto, quien escribe esta carta a Klein es el Winnicott analista y miembro de la institucionalidad psicoanalítica, que vislumbra los riesgos que la inflación de la técnica tiene sobre la práctica del psicoanálisis. Este llamado de atención será relevante para lo que pasaremos a examinar a continuación.

\section{Análisis, Discusión y Conclusión}

Aún estamos en la clínica con niños, pero a condición de discutir ahora una política de la infancia, es decir, cómo se conducen las instituciones mandatadas a enfrentar problemáticas de vulneración de derechos. Será interesante también averiguar cuál fue la posición de Winnicott frente 
a esta cuestión que, como se verá, es profundamente coherente con su manera de entender la clínica infantil.

Esta clínica de niños se convierte hoy día en una política de Estado, que imprime manuales donde nos explican cómo hay que orientarse para avalar una cierta política de infancia ya instituida, este es el caso de las Bases Técnicas del Servicio Nacional de Menores, las cuales aparecieron recientemente en nuestro país. Este documento está dirigido a los Programas de Protección Especializados (Sename, 2011) que intervienen con niños institucionalizados, con el fin de preparar su reintegración a familias adoptivas. En estas bases se detallan una serie de directrices que el profesional debe aplicar para conseguir el objetivo de un reacomodo del niño que resulte ser lo más "benigno" posible.

A fin de introducir nuestro debate, leamos algunas líneas de este manual, donde se deja traslucir, digámoslo así, la ideología actual que imparte el Estado chileno sobre infancia y adopción:

Desde esta comprensión, el terapeuta se ocupará de entrenar al niño/a a través de la siguiente secuencia de estrategias de regulación y autorregulación en momentos de estrés, las que se presentan según el grado de complejidad (de lo más simple y directo) y el grado de éxito (de acuerdo le sea de utilidad al niño): a) Búsqueda de un adulto presente cuando el niño/a esté estresado se le orientará a solicitar de manera verbal o implícita, una contención corporal al adulto mencionado: un abrazo, una caricia, palabras suaves y afectivas, o simplemente la compañía activa hasta que el estrés haya disminuido (Sename, 2011, p. 12). Se entiende bien que la concepción del niño trata de un individuo que podrá presentar emociones disruptivas que deben ser controladas dentro de cierto marco razonable, y se lo debe "entrenar" a fin de volverlo más "civilizado". También se entrena al adulto, el que debería seguir una pauta de afectos positivos para entregar al niño, a fin de volver a "regularlo”. La impresión es que este manual retrocede hacia una mecanización de los comportamientos, según lo que hemos estudiado, tan criticado por Winnicott, para llegar a desconocer todo lo que entendemos por subjetividad. ¿Qué consecuencias puede tener esta perspectiva sobre un niño que 
transita en una situación de tanta vulnerabilidad como lo es la adopción? Bajo el amparo de defender los derechos del niño y su salud mental, se lo ubica (y al terapeuta también) arbitrariamente en posición de objeto: es decir, algo que se puede tomar, entrenar, regular, reubicar, informarle sobre su historia, etc.

Esta posición terapéutica es bastante gráfica a propósito de las tergiversaciones que sufre el concepto de "objeto transicional" de Winnicott. En efecto, se pretende hacer de este una técnica de regulación y entrenamiento emocional del niño, para "tranquilidad" del adulto que no sabe bien qué hacer con él:

Recurrir a la contención emocional con un muñeco/a, peluche o juguete que sea de preferencia del nińo/a. Dado que resulta complejo garantizar que el niño/a pueda tener un juguete y acceder al mismo en momentos de estrés, se recomienda que el programa responsable de su intervención, sea quien lo provea, lo cual desde un punto simbólico puede resultar en el inicio del vínculo terapéutico. La idea es que el niño/a pueda comprender y tener la posibilidad de recurrir a su muñeco o peluche (pudiendo abrazarlo y tranquilizarse con este) cuando se encuentre estresado y no exista adulto responsable capaz de o disponible para contenerlo en ese momento. El principio que subyace a esta técnica es "ignorar-distraer y redirigir”, o sea, que el niño/a incorpore como estrategia de autorregulación el hecho de que cuando se sienta estresado y no exista regulación externa, pueda relocalizar su interés y su conducta en otras situaciones más positivas (Sename, 2011, p. 13).

Más aún entonces: se trata de "ignorar-distraer y redirigir" al niño, con ello se borra definitivamente la profundidad del pensamiento winnicottiano, que se "gastó" tanto en hacer comprender la ética del sujeto; porque, en definitiva, quién se preguntaría por ese niño que recurre $-y$ tal vez "regresivamente" - a ese objeto transicional, olvidado de su primera infancia, y le vuelve a dar vida, para tal vez él mismo conservarse con vida frente a la desertificación subjetiva que le impone un mundo adulto que mecaniza toda la dimensión de los cuidados del niño, entonces ¿̨hasta qué límites puede llevarse el paradigma de la psicología del aprendizaje? 
Otra complejidad que muestra el documento es aún más delicada, porque tiene que ver con el modo de tratar la historia del nińo, la que existía antes de la adopción. ¿Cuál será la historia "oficial” que se le "enseñará”? Veamos otra cita:

Asimismo, se deberá incorporar la técnica del libro "Mi historia" para los niños/as sobre los 7 años, para quienes se estima que les permitirá conocer aspectos significativos de su historia, que pudiera dar respuesta a algunas interrogantes que presente, como asimismo, favorecer la construcción de su identidad; no obstante, es preciso señalar que la técnica se deberá trabajar con un enfoque informativo y no con el objetivo de resignificar las experiencias dolorosas (Sename, 2011, p. 14).

La elaboración del libro será de responsabilidad del terapeuta del PRI, quien deberá darlo a conocer al niño/a si este es mayor de 7 años de edad, haciendo énfasis en los aspectos positivos de su vida y señalando la proyección que existe de que él/ella pueda integrarse a una familia alternativa a la de origen, cuando se prevea que esta posibilidad es factible, lo que estará determinado en gran parte por la edad y características del niño/a. Se espera que con posterioridad los niños/as puedan seguir trabajando esta técnica, apoyados por la familia donde se integrarán (Sename, 2011, p. 14).

Con niños/as menores de 7 años, debido a que en esta etapa del desarrollo evolutivo aún no pueden procesar y significar simbólicamente sus experiencias pasadas, deberán ser los adultos que asuman los roles parentales en la etapa de la integración familiar los responsables de trabajar esta técnica [la del libro "Mi historia"] con el niño/a (Sename, 2011, p. 13).

Este extracto de texto contiene el núcleo de lo que tratan estas directrices: el niño trae una historia de abandono parental y diversas clases de abusos que han vulnerado sistemáticamente sus derechos, esta historia -la historia como acontecimiento, no la historia subjetiva- se trabaja con el terapeuta de un modo "informativo", no elaborativo, para 
prepararlo a la reubicación en su nueva familia. Y la escritura del libro "Mi historia" será responsabilidad del terapeuta, entonces, ¿qué cabida se le da a la historia del niño?

El documento pretende resolver el problema sobre la transmisión de la historia desde la convicción que esta pudiera entrenarse, informarse, reeditarse. Este modo de ver las cosas recuerda el trabajo de los autores Abraham y Torok (2005), cuando trabajan el concepto de cripta, es decir, plantean que lo traumático deriva de un espacio en el psiquismo ni siquiera olvidado, es decir, un fragmento de historia "muerta", ya que no alcanzó el estatuto de inscripción como huella mnémica:

Para nosotros, analistas, es precisamente ese maquillaje, esa denegación la que dará prueba, más que nada, de la presencia de lo que vuelve como "realidad" para nuestro paciente, realidad a escamotear, se entiende (...) El concepto metapsicológico de Realidad remite, en el aparato psíquico, el lugar donde el secreto está sepultado (Abraham \& Torok, 2005, p. 227).

En nuestro lenguaje y en el contexto político de la historia reciente en nuestro país, se asemejaría a lo que conocimos y aún perdura como los desaparecidos, aquel fragmento de la "realidad" que no se supo sobre destino de muerte, prolongando un duelo que no cesa. Porque la historia colectiva es algo que también se la puede hacer desaparecer ${ }^{4}$.

Esta posibilidad de tener lugar-consideremos la interiorizaciónapropiación de la escena del niño winnicottiano jugando de espaldas a la madre, relevante para una clínica de lo traumático- requiere tiempo. No cualquier tiempo, sobre todo no el tiempo lineal o cronológico: en esto Winnicott es freudiano, porque resalta el tiempo subjetivo, que se constituye a posteriori \{après coup\}: "el terapeuta debe poder asimilar los conflictos del paciente, esto es, asimilarlos y aguardar a que su resolución se opere en el paciente mismo, antes que perseguir ansiosamente su cura” (Winnicott, 2008, p. 10). En efecto, para Winnicott el segundo tiempo es lo que constituye el recuerdo propiamente tal, es el que permite al paciente

4 Hay esfuerzos en Chile por visibilizar la infancia como punto crítico de una dimensión política sobre el modo de tratar la memoria histórica por el Estado. Es el caso del Colectivo Infancia y Política (CIP, 2012), espacio de discusión abierto sobre políticas públicas en la infancia. 
lograr una relación nueva con lo acontecido. Un pequeño artículo de Winnicott se dedica a este asunto, nos referimos a Notas sobre el factor tiempo en el tratamiento (Winnicott, 1998c), donde es muy crítico con aquellos intentos terapéuticos que pretenden acortar arbitrariamente el tiempo que necesita un paciente para hacer esta experiencia. Esto quiere decir que dejamos que ese saber pueda ser adquirido por nuestros propios pacientes en $s u$ tiempo. El trabajo de un psicoanálisis hace que lo pasado se actualice por la transferencia, y que incluso el inicio de un padecimiento, por ejemplo, una escena traumática, se retome de otro modo solo al final. En todo caso, lo que un analista tendría que cautelar es la apertura a un porvenir, que es otro modo de hablar del deseo: "Todos sabemos que cuando un paciente empieza a hablar de su pasado está al mismo tiempo empezando a hablar de su futuro" (Winnicott, 1998c, p. 273). En este sentido, la historia no puede estar excluida del niño, porque ella es a la vez la historia de los padres, esta historia el niño la escribe y reescribe en sus diversos jugares, por ejemplo, en la construcción de escenas al modo de fantasías que buscan tramitar preguntas que interrogan sobre su origen filial, pero para hacerse de una historia propia. Por eso resulta preocupante para el psicoanalista infantil encontrarse con un niño que no juega, juego en sentido de "playing" (y no "game"), es decir, donde el niño está implicado como sujeto abierto a un porvenir.

Esta violencia sobre la historia del niño, promovida por las directrices que establecen estas Bases Técnicas, finalmente nos obliga a pronunciarnos sobre una verdadera confusión de lenguajes (Ferenczi, 1966). Si queremos ahora referirnos al psicoanalista Sándor Ferenczi, en efecto, este autor nos invita a pensar a partir de esta conferencia escrita, que trata sobre los efectos de los abusos y la violencia en el niño, cómo es que un adulto puede acercarse al lenguaje del niño sin confundirse ni confundirlo. Porque explica, en el abuso el adulto confunde los deseos del niño con los suyos propios, en eso consiste su agresión. Muy cercano, y hasta precursor al pensamiento de Winnicott, este autor es bastante claro en afirmar que este problema no puede abordarse si no se está dispuesto a tomar en cuenta los sometimientos a los que pudiera estar "dispuesto" el niño para preservar su vínculo de amor con el adulto. Pero la consecuencia es el sacrificio de 
una parte del ser del niño. Desde este ángulo, es otra forma de violencia decir -como se afirma en estas Bases técnicas-, que niños menores de siete años "(...) aún no pueden procesar y significar simbólicamente sus experiencias pasadas" (Sename, 2011, p. 13). ¿No representará esta afirmación otra confusión del adulto, que niega la subjetividad del niño, para ubicarlo como un ente "externo" a él?

¿Cómo se habría pronunciado Winnicott sobre esta concepción del niño y sobre este modo de tratar la historia? Ya que este autor fue uno de los pocos analistas de su época -tal vez el primero- que intentó un diálogo fecundo entre el saber psicoanalítico y la institucionalidad que regía las políticas de infancia en la Inglaterra de los años cincuenta y sesenta. Para abordar la pregunta anterior habría que averiguar qué pensaba Winnicott sobre los problemas que surgen de la adopción. Introductoriamente, establece una mirada panorámica del tema:

(...) divido los problemas de la adopción en dos amplias categorías: en una están los problemas correspondientes simplemente al hecho de la adopción, que pueden estar presentes aunque no originen angustia; en la otra, las complicaciones resultantes del manejo deficiente del bebé antes de la adopción (Winnicott, 1998a, pp. 155-156).

En cualquier caso, la adopción, aunque puede llegar a ser una situación tremendamente satisfactoria para padres e hijos, siempre constituye un problema -no necesariamente una dificultad- que deben resolver la institución que ampara al niño, el niño mismo y los padres adoptivos, y más ampliamente todavía, las políticas de gobierno. Este problema principalmente tiene que ver con cómo estos padres pueden transmitir la dimensión del amor -cuestión infinitamente más relevante que los meros cuidados físicos- a un nińo que debe convertirse en hijo, pero que no ha nacido en el seno familiar. Para ello el trabajo de las instituciones (psicólogos, asistentes sociales, etc.) es fundamental en la tarea de poder anticipar a los padres las posibles dificultades que puedan encontrarse, dada la condición de abandono y deprivación del niño durante su primera infancia. No hay que olvidar que para Winnicott la cuestión del niño deprivado adquiere importancia en el psicoanálisis infantil, cuando el relato 
edípico aparecía como coordenada unívoca ofrecida a la interpretación del analista. Cuando los problemas propios de la adopción se suman a la negligencia o desconocimiento de las instituciones, los padres tendrán un caso y no un hijo, pudiendo agravar aún más las dificultades.

El compromiso de Winnicott con su realidad actual, que asume como su deber de psicoanalista "ciudadano", también se había manifestado cuando dedica sendos trabajos sobre la guerra y las consecuencias que este estado de excepción ocasiona en los niños, los cuales se hacen evidentes en sus relatos y dibujos. Por ejemplo, en su conferencia Los niños en la guerra (Winnicott, 2007b), sostiene que:

Solo es posible comprender las reacciones de los niños ante la difusión de las noticias de guerra estudiando en primer lugar, o por lo menos teniendo en cuenta, el mundo interior inmensamente rico de cada nińo, que constituye el trasfondo para todo lo que incide sobre él desde la realidad externa (Winnicott, 2007b, p. 80).

A continuación hablará sobre los niños que han perdido a sus padres en la guerra y que deben ser "reubicados" en otras familias, con las implicancias que ello trae para padres e hijos. Sobre todo le interesa la pregunta del cómo se constituyen los lazos de amor entre el niño y su familia, cuando la guerra amenaza con llevarse este sentimiento que apuntala la vida. Aquí entiende por amor la capacidad del niño para recuperar la confianza en que los nuevos padres puedan llegar a comprenderlo:

Ahora acudía a ustedes en busca de amor y de la sensación de ser amado. En esa nueva posición frente al niño, ustedes eran no solo las únicas personas que hacían algo por él, sino que también estaban allí para comprenderlo y ayudarlo a mantener vivo el recuerdo de su propia familia (Winnicott, 2007a, p. 93).

El amor en Winnicott habría que entenderlo como la disposición de la familia para dar un lugar al niño que lo aloje como hijo, situación que le permitiría apropiarse de una nueva historia que es también la historia de deprivación que hubo: de ahí que podrá hacerse la pregunta por su lugar en el deseo de estos nuevos padres. Pero al mismo tiempo que el amor es una apertura al sujeto, lo que Winnicott denomina agresión no hace menos: lejos del carácter negativo que se le dio desde cierto psicoanálisis -y 
obviamente también desde estas Bases Técnicas-, la agresión para Winnicott demuestra la fuerza vital y el impulso creador del sujeto. Aquí, agresión no es destrucción ni automutilación. Cada vez que en clínica encontramos una inhibición significativa en el nivel de la agresión, habrá que preguntarse por los diversos sometimientos, también actuantes en la transferencia, y en última instancia, por esa "fuerza vital" que subrayaba Winnicott.

Para ser un poco más ilustrativos: Winnicott nos presenta el caso de William, niño de cuatro años que fue adoptado al mes de nacer y que es traído por sus padres a consultar por presentar el síntoma de golpearse gravemente la cabeza contra la pared. Winnicott descubre que los padres aún se estaban recuperando de un aborto ocurrido antes de adoptar al chico, lo cual los hacía atravesar por intensos sentimientos de culpa. El relato del caso es como sigue: "Al principio me pareció que no existía ningún trastorno del desarrollo emocional, pero poco a poco se puso en evidencia en la consulta que ninguno de los padres guardaba recuerdos precisos de detalles de su infancia" (Winnicott, 1998d, p. 170). En esta entrevista encuentra que la clave del problema consistía en una dificultad de los padres para establecer con el chico una-como se expresa Winnicott"buena relación":

La madre estaba lidiando con su culpa por el aborto y también con el resentimiento por el hecho de que no fuera hijo suyo. El padre, imprevistamente, sintió una profunda repulsión cuando vio al bebé. Como consecuencia de esto, lo cuidaron bien físicamente, pero durante un período que quizás duró un año no fue realmente amado; y por cierto al principio la madre no tuvo, con relación a las necesidades del niño, esa orientación especial que es natural en una madre con un bebé propio (Winnicott, 1998d, p. 172).

Este breve relato sobre adopción muestra los estragos a los que puede llevar una mecanización en los cuidados del niño, cuando no toma en cuenta el hecho que estos padres estaban interferidos por el duelo de no haber podido cumplir con su deseo de convertirse en padres "naturales", y del sentimiento de culpa por un aborto anterior y quizás también por no haber podido hablar sus dificultades en esta adopción. El efecto terapéutico de la consulta con Winnicott se produce por su escucha: en vez de buscar 
orientación en algún saber "oficial" sobre infancia y adopción, resuelve dar lugar a la singularidad del caso, es decir, a la dimensión de lo olvidado de la historia de estos padres y por haberles facilitado la comprensión de que ninguna adopción puede pensarse en términos de ideales. Mejor es pensarla en términos de posibilidades.

A modo de conclusión, diremos que hemos comenzado este ensayo con la explicitación de una dificultad, la de una transmisión de la clínica winnicottiana, y en términos más amplios, del pensamiento de Winnicott, y hemos cerrado con una posibilidad: la de volver a leer a Winnicott para ayudarnos a situar una posición ética que nos permita debatir con mejor lucidez las nuevas políticas sobre infancia y adopción en nuestro país.

Debe poder discutirse el impacto que significa la implantación de este tipo de políticas, por las implicancias que puede traer la importación de ciertas formas de pensar la clínica que a juicio de algunos -me incluyocorre el riesgo de excluir el campo subjetivo y subjetivante, que en una problemática de adopción se nos revela como trascendente para el destino del niño y su familia, ¿no es esta una forma velada de violencia sobre el niño, amparada en políticas de "protección" de la infancia? Hay que ver cuánto se aleja el pensamiento de Winnicott de estas políticas, cuando su ética lo lleva a proponer al nińo como una subjetividad actuante y no como un objeto respondiente o entrenable.

La dificultad para la transmisión de una realidad subjetiva que se distancia radicalmente de los meros cuidados físicos o psíquicos del niño no debe ser excusa para abandonar ese esfuerzo, sobre todo si nos guiamos por lo que hemos llamado aquí una ética winnicottiana, es decir, el testimonio que nos dejó este autor sobre su deseo: el de hacer resistencia a aquellas posturas teóricas de su época sobre un psicoanálisis -es el caso particular del "kleinismo"-, que en su discursividad había minimizado la cuestión del sujeto, del lugar del otro en la constitución subjetiva y de la realidad de los contextos sociales y políticos, además de haber perdido toda originalidad en su escritura. La "exhumación" de esa discusión tiene ecos de actualidad para iluminar hoy día los elementos más controversiales de las Bases Técnicas distinguidos aquí. 


\section{Referencias}

Abraham, N. \& Torok, M. (2005). La Corteza y el núcleo. Buenos Aires: Amorrortu.

Colectivo Infancia y Política (CIP). (2012). Recuperado el 17 de agosto de 2012 de http://infanciaypolitica.wordpress.com

Ferenczi, S. (1966). La confusión de lenguajes entre los adultos y el nińo. En S. Ferenczi, Problemas y métodos del psicoanálisis (pp. 139-149). Buenos Aires: Hormé.

González, M. (2010). Los tiempos en la infancia: Desde la alucinación hacia la posibilidad de recuerdo. En Espacios de tiempo. Clínica de lo traumático y procesos de simbolización (pp. 57-67). Santiago: Colección Praxis Psicológica, Universidad de Chile.

Klein, M. (2004). Nuestro mundo adulto y sus raíces en la infancia. En M. Klein, Obras completas 3 (pp. 251-267). Buenos Aires: Paidós.

Lacan, J. (1967-68). El acto analítico (1967-8). Manuscrito no publicado.

Lacan, J. (2003). El estadio del espejo como formador de la función del yo [je] tal como se nos revela en la experiencia psicoanalítica En J. Lacan, Escritos I (pp. 86-93). Buenos Aires: Siglo XXI.

Rodulfo, R. (2009). Trabajos de la lectura, lecturas de la violencia. Lo creativo-lo destructivo en el pensamiento de Winnicott. Buenos Aires: Paidós.

Servicio Nacional de Menores (2011). Bases técnicas, Duodécima Convocatoria de Concurso Público de Proyectos para la línea de acción Programas de Protección Especializados Modalidad: Programa de Intervención con niños/as Institucionalizados y su preparación para la Integración a Familia Alternativa a la de Origen (PRI). Recuperado el 1 de marzo de 2012 de http://www.sename.cl/wsename/licitaciones/p12_19-07-2011/Bases_TEC_PRI.pdf

Winnicott, D. (1990). El gesto espontáneo. Barcelona: Paidós. (Trabajo original publicado en 1987).

Winnicott, D. (1991a). Sobre "El uso de un objeto". En D. Winnicott, Exploraciones psicoanalíticas I (pp. 261-293). Buenos Aires: Paidós.

Winnicott, D. (1991b). Sobre las bases del self en el cuerpo. En D. Winnicott, Exploraciones psicoanalíticas I (pp. 311-335). Buenos Aires: Paidós.

Winnicott, D. (1993). Un modo personal de ver el aporte kleiniano. En D. Winnicott, Los procesos de maduración y el ambiente facilitador (pp. 223-233). Buenos Aires: Paidós.

Winnicott, D. (1994). La cura. En D. Winnicott, El hogar, nuestro punto de partida (pp. 130-140). Buenos Aires: Paidós. 
Winnicott, D. W. (1998a). Dos niños adoptados. En D. Winnicott, Acerca de los niños (pp. 155-156). Buenos Aires: Paidós.

Winnicott, D. (1998b). La etiología de la esquizofrenia infantil en términos de la falla adaptativa. En D. Winnicott, Acerca de los niños (pp. 259-264). Buenos Aires: Paidós.

Winnicott, D. (1998c). Notas sobre el factor tiempo en el tratamiento. En D. Winnicott, Acerca de los niños (pp. 271-274). Buenos Aires: Paidós.

Winnicott, D. (1998d). Obstáculos en la adopción. En D. Winnicott, Acerca de los niños (pp. 167-174). Buenos Aires: Paidós.

Winnicott, D. (2007a). El niño evacuado. En D. Winnicott, El niño y el mundo externo (pp. 89-93). Buenos Aires: Ediciones Hormé.

Winnicott, D. (2007b). Los niños en la guerra. En D. Winnicott, El niño y el mundo externo (pp. 75-80). Buenos Aires: Ediciones Hormé.

Winnicott, D. (2007c). Realidad y juego. España: Editorial Gedisa.

Winnicott, D. (2008). Clínica psicoanalitica infantil. Buenos Aires: Hormé.

Fecha de recepción: 15 de marzo de 2012.

Fecha de aceptación: 24 de mayo de 2012. 